\title{
Analysis of Negative Bias Temperature Instability in Body-Tied Low-Temperature Polycrystalline Silicon Thin-Film Transistors
}

\author{
Chih-Yang Chen, Student Member, IEEE, Ming-Wen Ma, Wei-Cheng Chen, Hsiao-Yi Lin, \\ Kuan-Lin Yeh, Shen-De Wang, and Tan-Fu Lei
}

\begin{abstract}
Negative bias temperature instability (NBTI) degradation mechanism in body-tied low-temperature polycrystalline silicon thin-film transistors (LTPS TFTs) is analyzed by the chargepumping (CP) technique. The properties of bulk trap states (including interface and grain boundary trap states) are directly characterized from the $\mathrm{CP}$ current. The increase of the fixed oxide charges is also extracted, which has not been quantified in previous studies of NBTI degradation in LTPS TFTs. The experimental results confirm that the NBTI degradation in LTPS TFTs is caused by the generation of bulk trap states and oxide trap states.
\end{abstract}

Index Terms-Charge-pumping (CP) technique, lowtemperature polycrystalline silicon thin-film transistors (LTPS TFTs), negative bias temperature instability (NBTI).

\section{INTRODUCTION}

L OW-TEMPERATURE polycrystalline silicon thin-film transistors (LTPS TFTs) are attracting much research interest as potential candidates for the realization of system on panel (SOP). For driving circuit operation, LTPS TFTs must be designed using the CMOS inverter configuration. During operation, p-channel TFT will be subjected to negative bias temperature instability (NBTI) when the input is at a low voltage level and the output is at a high voltage level. In MOSFETs, it is well accepted that the NBTI degradation originates from the breaking of the hydrogenated silicon $(\mathrm{Si}-\mathrm{H})$ bonds, resulting in the generation of interface and oxide trap states [1]-[3]. However, in LTPS TFTs, due to the existence of trap states in the grain boundaries and at the poly-Si/SiO ${ }_{2}$ interface, more $\mathrm{Si}-\mathrm{H}$ bonds may be found in the channel region than with MOSFETs. Further, due to the poor thermal conductivity of the glass substrate and high operating voltage, NBTI can be an important reliability issue for LTPS TFTs. Therefore, it is important to study NBTI behaviors and the related degradation mechanisms of LTPS TFTs.

Manuscript received September 14, 2007; revised November 9, 2007. This work was supported by the National Science Council, Taiwan, R.O.C., under Contract NSC95-2221-E-009-279. The review of this letter was arranged by Editor J. Sin.

C.-Y. Chen, M.-W. Ma, S.-D. Wang, and T.-F. Lei are with the Institute of Electronics, National Chiao Tung University, Hsin-Chu 300, Taiwan, R.O.C. (e-mail: cyc.ee92g@nctu.edu.tw; william.ee94g@nctu.edu.tw; bryant519@ yahoo.com.tw; tflei@cc.nctu.edu.tw).

W.-C. Chen, H.-Y. Lin, and K.-L. Yeh are with the Toppoly Optoelectronics Corporation, Chu-Nan 350, Taiwan, R.O.C. (e-mail: weicheng@toppoly.com; hylin2@toppoly.com; klyeh@toppoly.com).

Color versions of one or more of the figures in this letter are available online at http://ieeexplore.ieee.org.

Digital Object Identifier 10.1109/LED.2007.914083

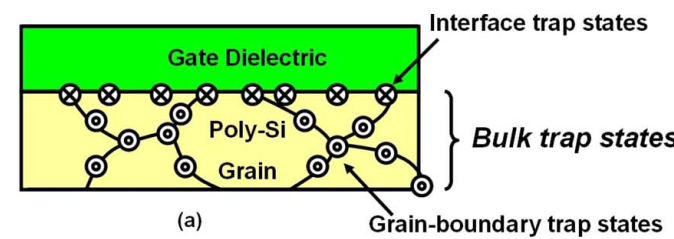

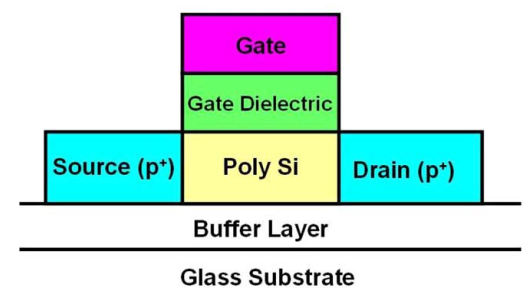

(b)

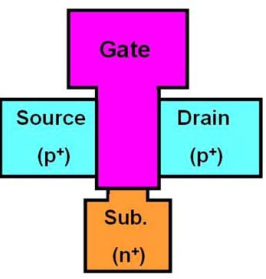

(c)
Fig. 1. (a) Schematic cross-sectional view of the critical poly-Si/ $/ \mathrm{SiO}_{2}$ interface showing interface trap states and grain boundary trap states. (b) Crosssectional and (c) top views of the LTPS TFT used in this letter.

NBTI has been reported to degrade the channel film of LTPS TFTs [4]. In previous studies, the channel properties have been found to be derived from the current-voltage characteristics. However, the channel current is affected through the potential barrier formed by the trap states [5]. Therefore, the bulk trapstate density $\left(N_{\mathrm{bulk}}\right)$ derived from the channel current does not indicate the real bulk trap-state density, but the effective bulk trap-state density. Koyanagi and coworkers [6], [7] and Balasinski and coworkers [8]-[10] proposed the charge-pumping (CP) technique to directly characterize the bulk trap properties of poly-Si TFTs. Unlike in MOSFETs, in LTPS TFTs, the grain boundary trap states also give rise to the $\mathrm{CP}$ current $\left(I_{\mathrm{CP}}\right)$. Consequently, $N_{\text {bulk }}$ as measured by the CP technique consists of both the grain boundary and interface trap states, as shown in Fig. 1(a). $N_{\text {bulk }}$ can be revealed from $I_{\mathrm{CP}}$ because carriers are observed as the generation-recombination current. As a result, the influence of $N_{\text {bulk }}$ is directly evaluated. However, use of this technique to analyze the NBTI effect in LTPS TFTs has not been reported. Given this, the aim of our study is to use the CP technique to investigate the behavior of $N_{\text {bulk }}$ during NBTI stress. Further, the role of oxide trap-state generation in the NBTI degradation mechanism is also identified.

\section{EXPERIMENT}

P-channel LTPS TFTs $(\mathrm{W} / \mathrm{L}=10 \mu \mathrm{m} / 10 \mu \mathrm{m})$ were fabricated on glass substrates. A 40-nm-thick amorphous Si layer was deposited by plasma enhanced chemical vapor deposition 


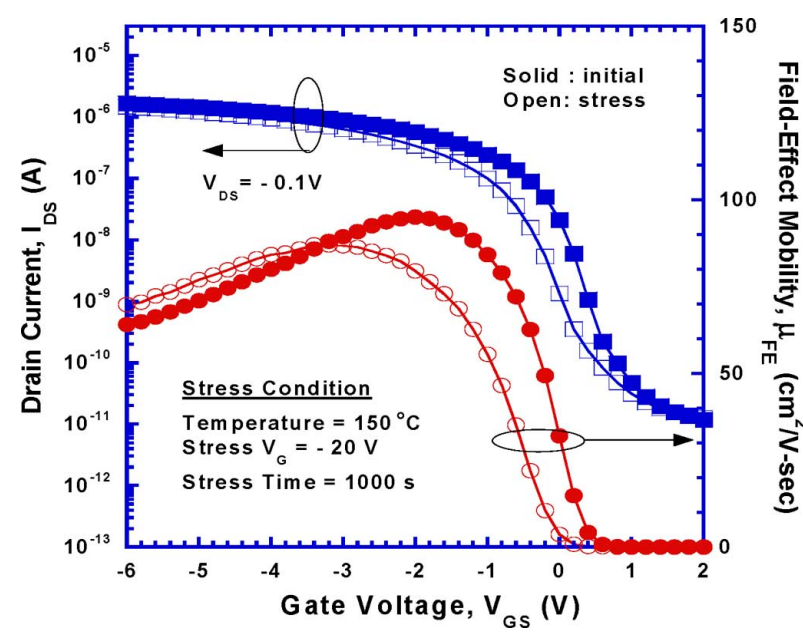

Fig. 2. Transfer characteristics of the LTPS TFT before and after $1000 \mathrm{~s}$ NBTI stress at $150{ }^{\circ} \mathrm{C}$ with the stress voltage of $-20 \mathrm{~V}$.

(PECVD) on a buffer layer and crystallized into poly-Si film by excimer laser annealing. Ion implantation was performed on the extra body terminal to form the heavily doped $\mathrm{n}^{+}$region. A 100-nm-thick $\mathrm{SiO}_{2}$ layer was deposited as the gate dielectric followed by Mo deposition as the gate. After source/drain formation, hydrogenation was performed with $\mathrm{NH}_{3}$ treatment. Then, the interlayer dielectric was deposited and densified, and the dopants were activated during this step. Finally, interconnection metal was deposited and patterned. The schematic cross sectional and top views of the device are shown in Fig. 1(b) and $1(\mathrm{c})$, respectively.

The NBTI stress was performed at the temperature ranging from $75^{\circ} \mathrm{C}$ to $150{ }^{\circ} \mathrm{C}$, and a gate voltage in the range of -13 to $-20 \mathrm{~V}$ was applied with the source, drain, and body grounded. During the CP measurement, a pulse train with frequency of $100 \mathrm{kHz}$ and fixed pulse amplitude of $1.5 \mathrm{~V}$ were applied to the gate while $I_{C P}$ was measured between the source/drain and substrate contact, and the base voltage was varied to tune the surface condition from inversion to accumulation.

\section{RESULTS AND DISCUSSION}

Fig. 2 shows the transfer characteristics of the LTPS TFT before and after the NBTI stress. The device shows degradation in the subthreshold swing and field-effect mobility, indicating that interface trap states were generated [11]. Further, the threshold voltage $\left(V_{\mathrm{th}}\right)$ shifts to the negative direction after the stress. Degradation can be attributed to the generation of bulk trap states and oxide trap states. Detailed analysis of these trap-state generations will be discussed later.

The inset of Fig. 3 shows the $I_{\mathrm{CP}}$ of the device before and after $1000 \mathrm{~s}$ NBTI stress. The increase in $I_{\mathrm{CP}}$ indicates that bulk trap states are generated during NBTI stress. Further, the $I_{\mathrm{CP}}$ curve shifts to the negative direction, implying that a net positive charge is clearly generated in the oxide or/and at the interface. Fig. 3 shows the time dependence of the $N_{\text {bulk }}$ generation $\left(\Delta N_{\text {bulk }}\right)$, which is calculated from $I_{\mathrm{CP}} . \Delta N_{\mathrm{bulk}}$ follows a power law dependence on the stress time with an exponent factor of $0.37-0.42$, implying that diffusion-controlled electrochemical reactions may take part in the process [12], [13].

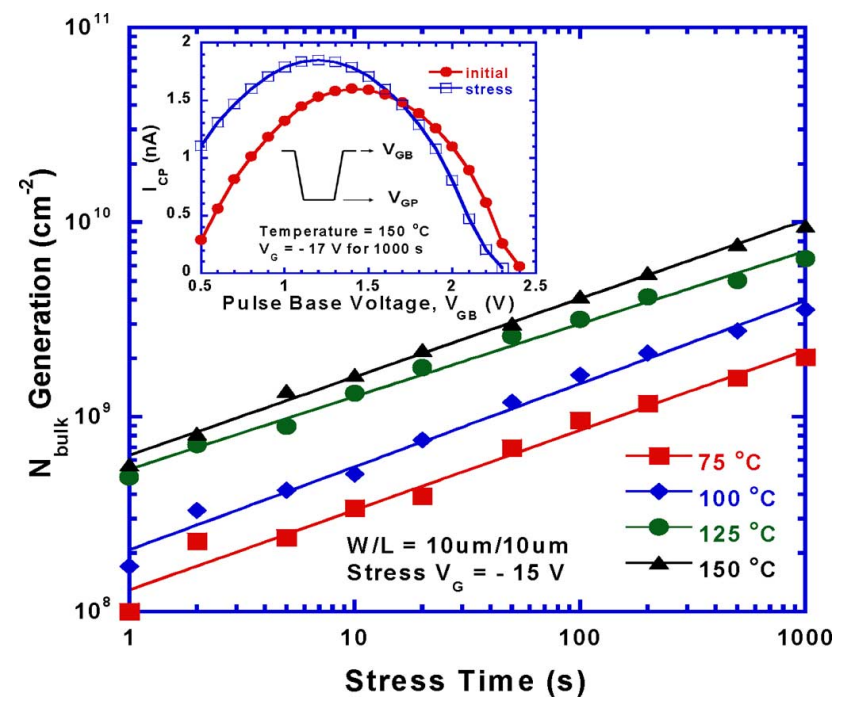

Fig. 3. Time dependence of the bulk trap-state density generation under various stress conditions. The inset shows the $I_{\mathrm{CP}}$ before and after $1000 \mathrm{~s}$ NBTI stress.
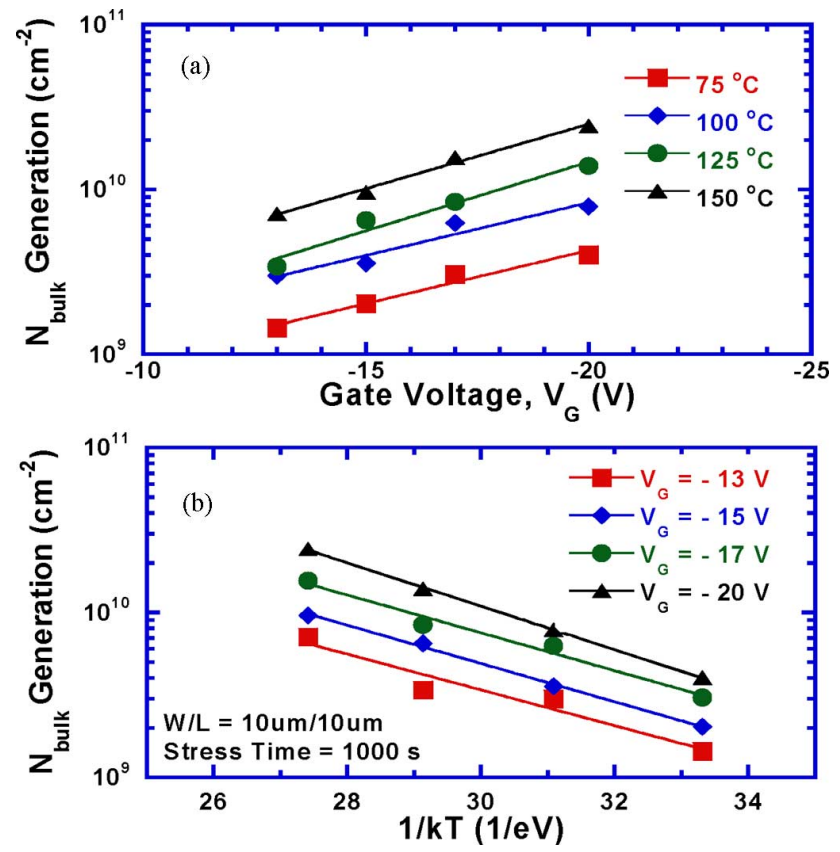

Fig. 4. Dependences of bulk trap-state density generation on the (a) stress voltage and (b) stress temperature of the LTPS TFTs under various stress conditions.

Further, $\Delta N_{\text {bulk }}$ also exhibits an exponential dependence on the stress voltage $\left(V_{G}\right)$ and the reciprocal of temperature $(1 / T)$. The experimental results show that the $N_{\text {bulk }}$ variation can be expressed as

$$
\Delta N_{\text {bulk }} \propto t^{n} e^{\left(-E_{a} / k T\right)} e^{C\left|V_{G}\right|} .
$$

The parameter $C$ extracted from Fig. 4(a) is between 0.14 and 0.19 , which is dependent on the process [14]. The activation energy $\left(E_{a}\right)$ extracted from Fig. $4(\mathrm{~b})$ is between 0.25 and $0.30 \mathrm{eV}$. $\Delta N_{\text {bulk }}$ increases with the stress voltage or temperature, implying that $N_{\text {bulk }}$ generation can be electrically and thermally activated. On the other hand, the charge-trapping model is unable 


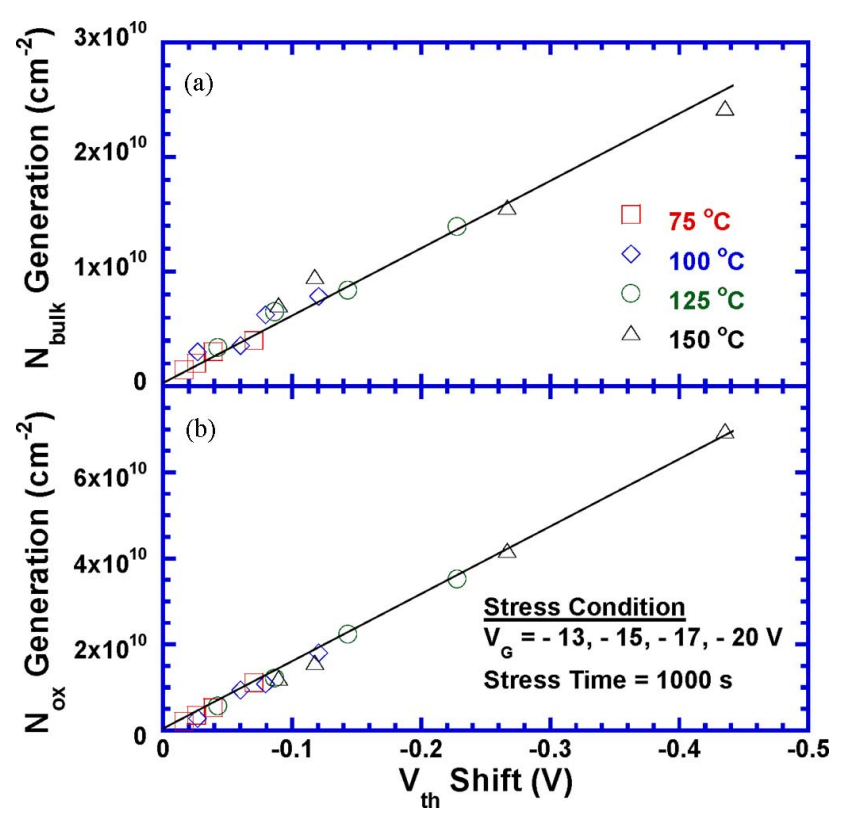

Fig. 5. Correlation between the increases of the (a) bulk trap-state density, (b) oxide trap-state density, and the threshold voltage shift.

to explain the exponential dependence of the $V_{\mathrm{th}}$ shift $\left(\Delta V_{\mathrm{th}}\right)$ on $V_{G}$ and $1 / T$ [15], [16]. Furthermore, the electric field across the gate dielectric (below $2 \mathrm{MV} / \mathrm{cm}$ ) was not high enough to cause hole injection. Therefore, we thus provide further confirmation that the NBTI degradation in LTPS TFTs is attributable to diffusion-controlled electrochemical reactions instead of the charge-trapping model.

Fig. 5(a) presents the correlation between $\Delta N_{\text {bulk }}$ and $\Delta V_{\text {th }}$, in which both these two physical quantities show a linear correlation. Because the degradation can be explained by the diffusioncontrolled electrochemical reactions [12], [13], we can assume the $\Delta V_{\mathrm{th}}$ is caused by the generation of $N_{\mathrm{bulk}}$ and oxide trap-state density $\left(N_{\text {ox }}\right)$. Therefore, the threshold voltage shift $\left(\Delta V_{\mathrm{th}}\right)$ may simply expressed as

$$
\Delta V_{\mathrm{th}}=-\frac{q\left(\Delta N_{\mathrm{bulk}}+\Delta N_{\mathrm{ox}}\right)}{C_{\mathrm{ox}}} .
$$

According to (2), $\Delta N_{\text {ox }}$ can be calculated from the measured $\Delta V_{\text {th }}$ and $\Delta N_{\text {bulk. }}$. Fig. 5(b) shows the correlation between $\Delta N_{\text {ox }}$ and $\Delta V_{\text {th }}$. We can conclude that $\Delta N_{\text {bulk }}$ alone cannot explain the measured $\Delta V_{\mathrm{th}}$ and that $\Delta N_{\text {ox }}$ must be taken into account.

By expanding the model proposed for bulk-Si MOSFETs [17], we introduce a model to explain the NBTI degradation mechanism for LTPS TFTs. The Si dangling bonds in the bulk channel region are assumed to be initially passivated by hydrogen atoms. During NBTI stress, the hydrogen atoms react with the holes and dissociate from the $\mathrm{Si}$ atoms, resulting in the generation of bulk trap states. The released hydrogen species diffuse or drift into the gate oxide and react with it, forming $\mathrm{OH}$ groups bounded to oxide $\mathrm{Si}$ atoms and leaving positive oxide trap states in the gate oxide. Finally, the hydrogen species diffuse into the gate oxide, becoming the reaction-limiting factor.

\section{CONCLUSION}

For the first time, the CP technique is utilized to analyze the NBTI degradation mechanism in LTPS TFTs. The properties of $N_{\text {bulk }}$ can be directly characterized from $I_{\mathrm{CP}}$. In addition, the increase of $N_{\text {ox }}$ is also extracted. Our results show that both $\Delta N_{\text {bulk }}$ and $\Delta N_{\text {ox }}$ are closely related to $\Delta V_{\mathrm{th}}$. This further confirms that $\Delta N_{\text {bulk }}$ alone cannot explain the measured $\Delta V_{\mathrm{th}}$ and that $\Delta N_{\text {ox }}$ must be taken into account. On the other hand, experimental results show that NBTI degradation can be electrically and thermally activated. Therefore, the operating voltage and power consumption have to be carefully designed, and new processes must be developed to suppress NBTI degradation and realize SOP.

\section{REFERENCES}

[1] C. E. Blat, E. H. Nicollian, and E. H. Poindexter, "Mechanism of negativebias-temperature instability," J. Appl. Phys., vol. 63, pp. 1712-1720, 1991.

[2] D. K. Schroder and J. A. Babcock, "Negative bias temperature instability: Road to cross in deep submicron silicon semiconductor manufacturing," J. Appl. Phys., vol. 94, pp. 1-18, 2003.

[3] A. Scarpa, D. Ward, J. Dubois, L. V. Marwijk, S. Gausepohl, R. Campos, K. Y. Sim, A. Cacciato, R. Kho, and M. Bolt, "Negative-bias temperature instability cure by process optimization," IEEE Trans. Electron Devices, vol. 53, no. 6, pp. 1331-1339, Jun. 2006.

[4] S. Maeda, S. Maegawa, T. Ipposhi, H. Nishimura, T. Ichiki, J. Mitsuhashi, M. Ashida, T. Muragishi, and T. Nishimura, "Negative bias temperature instability in poly-Si TFTs," in VLSI Symp. Tech. Dig., 1993, pp. 29-30.

[5] L. Levinson, F. R. Shephred, P. J. Scanlon, W. D. Westwood, G. Este, and M. Rider, "Conductivity behavior in polycrystalline semiconductor thin film transistors," J. Appl. Phys., vol. 53, pp. 1193-1202, 1982.

[6] M. Koyanagi, I.-W. Wu, A. G. Lewis, M. Fuse, and R. Bruce, "Evaluation of polycrystalline silicon thin film transistors with the charge-pumping technique," in IEDM Tech. Dig., 1990, pp. 34.6.1-34.6.4.

[7] M. Koyanagi, Y. Baba, K. Hata, I.-W. Wu, A. G. Lewis, M. Fuse, and R. Bruce, "The charge-pumping technique for grain boundary trap evaluation in polysilicon TFT's," IEEE Electron Device Lett., vol. 13, no. 3, pp. 152-154, Mar. 1992.

[8] A. Balasinski, J. Worley, M.Zamanian, and F. T. Liou, "Reference voltages and their stress-induced changes in thin film transistors as determined by charge pumping," in IEDM Tech. Dig., 1995, pp. 529-532.

[9] A. Balasinski, J. Worley, K. W. Huang, J. Walters, and F. T. Liou, "Observation of two types of trapping centers in thin film transistors using charge pumping technique," IEEE Electron Device Lett., vol. 16, no. 10, pp. 460-462, Oct. 1995.

[10] A. Balasinski, J. Worley, K. W. Huang, and F. T. Liou, "Identification of $\mathrm{Si} / \mathrm{SiO}_{2}$ interface properties in thin film transistors with charge pumping technique," J. Electrochem. Soc., vol. 142, pp. 2717-2721, 1995.

[11] T.-J. King, M. G. Hack, and I.-W. Wu, "Effective density-of-states distributions for accurate modeling of polycrystalline-silicon thin-film transistors," J. Appl. Phys., vol. 75, pp. 908-913, 1994.

[12] S. Ogawa and N. Shiono, "Generalized diffusion-reaction model for the low-field charge-buildup instability at the $\mathrm{Si}_{-} \mathrm{SiO}_{2}$ interface," Phys. Rev. $B$, vol. 51, pp. 4218-4230, 1995.

[13] S. Ogawa, M. Shimaya, and N. Shiono, "Interface-trap generation at ultrathin $\mathrm{SiO}_{2}$ (4-6 nm)-Si interfaces during negative-bias temperature aging," J. Appl. Phys., vol. 77, pp. 1137-1148, 1995.

[14] A. T. Krishnan, V. Reddy, and S. Krishnan, "Impact of charging damage on negative bias temperature instability," in IEDM Tech. Dig., 2001, pp. 865868 .

[15] M. J. Powell, C. V. Berkel, and J. R. Hughes, "Time and temperature dependence of instability mechanisms in amorphous silicon thin-film transistors," Appl. Phys. Lett., vol. 54, pp. 1323-1325, 1989.

[16] N. D. Young and A. Gill, "Electron trapping instabilities in polycrystalline silicon thin film transistor," Semicond. Sci. Technol., vol. 5, pp. 72-77, 1990.

[17] K. O. Jeppson and C. M. Svensson, "Negative bias stress of MOS devices at high electric fields and degradation of MNOS devices," J. Appl. Phys., vol. 48, pp. 2004-2014, 1977. 